\title{
An examination of the sensitivity of successive discrimination reversal (SDR) measures to differences in motivational level with squirrel monkeys ${ }^{1,2}$
}

\author{
ROBERT L. GOSSETTE AND JEROME FELDMAN, \\ COMPARATIVE ANIMAL BEHAVIORAL LABORATORIES, \\ HOFSTRA UNIVERSITY, Hempstead, L. I., N. Y. 11550
}

To determine the sensitivity of successive discrimination reversal (SDR) measures to differences in motivational level, performances of two groups of Squirrel monkeys on a spatial reversal were compared at $10 \%$ and $30 \%$ body weight reduction values. Greater error scores were made by the $30 \%$ group. As in previous work with pigeons, the locus of the motivational effect appeared to be increased resistance to extinction of the previously correct response.

Detailed comparisons of SDR performances of a number of avian and mammalian species have yielded consistent inter-species differences that have both taxonomic and phyletic significance (Gossette, 1966). For example, phyletically "higher" species display greater error reduction across reversal problems than do the "lower" organisms. In addition inter-species differences are smallest for those species separated by the smallest taxonomic distance and are greatest for taxonomically remote species (Gossette, Gossette, \& Riddell, 1966). Thus the use of SDR methodology to calibrate inter-species performance differences would appear to be promising. However, one serious criticism of such comparisons is that the obtained differences may reflect failure or inability to equate such methodological variables as motivational or incentive levels across species, and not some more basic dimensional difference, e.g., "learning ability." A useful test of the validity of such criticism would be to determine whether or not SDR measures are sensitive to variation of these variables.

In a recent study (Gossette \& Hood, 1967) increase in motivational level was shown to heighten errors, especially initial errors, by pigeons on a spatial SDR task during the first five problems of reversal training. However, this effect gradually disappeared over the next few problems. As a further extension of this line of inquiry a similar investigation was conducted to see if variation in motivational level could influence error scores of Squirrel monkeys on a spatial SDR task.

Method. All training and measurement of reversal performance was conducted in a form-board box, previously described (Gossette \& Inman, 1966). For this study, a spatial discrimination requiring a choice of one of two round white blocks to obtain a food reward was successively reversed for 19 problems.

Eight adult Squirrel monkeys obtained from local suppliers served as Ss. An attempt was made to acquire fully mature Ss. The mean weight at the beginning of testing was $832 \mathrm{~g}$, with a range from $555 \mathrm{~g}$ to $1050 \mathrm{~g}$. Following determination of ad lib feeding weights, four Ss were randomly assigned to a $10 \%$ weight reduction condition while the remaining Ss were assigned to a $30 \%$ weight reduction condition. Conventional primate laboratory chow was supplemented by fresh fruits and vegetables daily. As each $S$ attained its assigned weight reduction value, it was habituated to the test chamber and trained to dislodge stimulus blocks to obtain a mealworm from the underlying foodwells. A series of preference testing sessions and forced responding sessions were provided to equalize response tendencies to both block positions. Then a position habit was established, Problem 1, with responding to the nonpreferred side reinforced. When a criterion of two or fewer errors on a given day was achieved, the reinforcement contingencies were reversed, with training continued until criterion was reached again. In this fashion, 19 reversals were obtained. One session of 20 trials was given daily. Noncorrection was employed. Reinforcement consisted of two mealworms. Late in the experiment one $S$ of both groups abruptly ceased to respond for mealworms, and was switched to $1 / 4$ of a grape. The data for these Ss did not deviate from that of their respective groups.

Results. Analysis of cumulative errors across the 20 problems by the Kruskal-Wailis test indicated that the $30 \%$ group produced significantly $(p<.02)$ more errors than the $10 \%$ group did. Unlike the previous study with pigeons, the heightened errors for the $30 \%$ group was least apparent during the first block of five problems, Fig. 1, and increased over the remaining problems. A similar analysis of sessions per problem yielded the same pattern of differences. As a check upon the extent to which the body weight reduction values chosen represented different levels of motivation, the response latencies for the two groups were compared across problems. Consistent with reports of this measure previously made (Hall, 1966) the 30\% group exhibited significantly $(\mathrm{p}<.05)$ shorter latencies.

In the earlier study with pigeons, the major locus of action of the difference in motivational level appeared to be in heightened initial errors (an abbreviated measure of resistance to extinction) for the $30 \%$ group. To some extent the same conclusion applies to the present data. The initial errors for the $30 \%$ group were somewhat greater than those for the $10 \%$ group. However, the difference was not significant. A better extinction measure is provided by the per cent incorrect on the first session of each problem. In this case, the $30 \%$ group produced significantly $(p<$ .05) more errors on each first session than did the 10\% group. Thus, as in the pigeon study, it appears that difference in motivation level exerts its principal effect through control over resistance to extinction of the previously correct response.

Discussion. It is increasingly apparent that motivational level can influence SDR measures. While the major effect was restricted to the early SDR problems in a study with pigeons, the opposite trend appeared to be true with the Squirrel monkeys in this study. Whether this discrepancy represents a species or a methodological factor remains to be determined. In any event a serious complication arises for those investigators interested in attempting inter-species comparisons with methodology such as SDR and by extension, learning set. Since SDR measures may vary with motivational level, it will be necessary to determine the range of variation for each species involved in inter-species comparisons. Obviously such considerations complicate the evaluation of inter-species SDR performance differences, and possibly reduce the value and certainly the economy of such behavioral measures in establishing a behavioral taxonomy of phyletic significance. However, in view of the present absence of a more promising alternative methodology, it appears that future investigation of inter-species SDR performance differences is strongly justified.

Perhaps the most econmical approach would be an experimental design in which inter-species SDR performances among both taxonomically remote and closely related species are obtained at several levels of motivation and incentive. The fragmentary evidence available to date (Gossette, Riddell, \& Gossette, 1966) would suggest that as the degree of taxonomical remoteness increases, the extent to which error functions of different species will overlap will decrease. However, even among relatively closely related species the magnitude of the motivational effect may not obscure inter-species separation. For example, the spatial SDR performance of Capuchin monkeys tested under $22 \mathrm{~h}$ food deprivation, Fig. 1, yielded an error function uniformly lower than both groups of Squirrels. None of the individual Capuchin error functions overlapped those of the individual Squirrel monkeys.

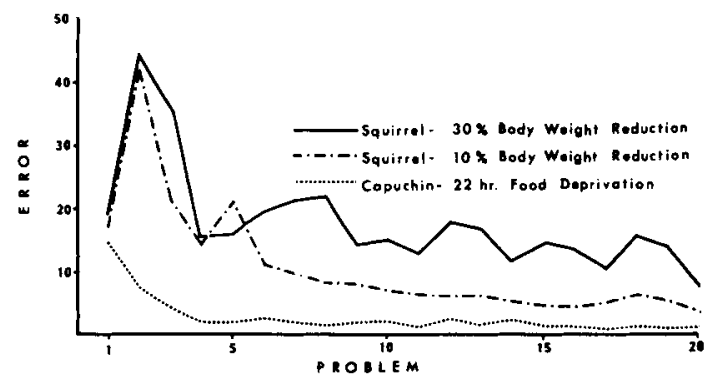

Fig. 1. Mean error per problem for Squinel monkeys across 19 reversals of a spatial discrimination as a function of level of food deprivation. 


\section{REFERENCES}

GOSSETTE, R. L. Comparison of successive discrimination reversal performances across 14 different avian and mammalian species. Amer. Zoologist, 1966, No. 4 (Abs).

GOSSETTE, R. L., GOSSETTE, MADELEINE, \& RIDDELL, W. Comparisons of successive discrimination reversal performances among closely and remotely avian species. Anim. Behav., 14, 560-564, 1966.

GOSSETTE, R. L., \& HOOD, PATRICIA. Successive discrimination reversal measures as a function of variation of motivational and incentive levels. Percept, mot. Skills, in press, 1968.
GOSSETTE, R. L. \& INMAN, N. Comparison of spatial successive discrimination reversal performances of two groups of New World monkeys. Percept. mot. Skills, 23, 169-170, 1966.

HALL, J. F. The psychology of learning. Philadelphia: J. P. Lippincotì, 1966.

\section{NOTES}

1. Supported in part by a grant No. GB-4799 from NSF awarded to the senior author.

2. We wish to thank Mr. Philip Slonim for his assistance in gathering some of the data reported in this study. 